\title{
Near-Optimal Algorithm for Self-configuration of Ad-hoc Wireless Networks
}

\author{
Sung-Eok Jeon and Chuanyi Ji \\ Georgia Tech, Atlanta GA 30332, USA
}

\begin{abstract}
To quantify the goodness of a configuration, we develop a probabilistic model of network configuration. A probabilistic graph then represents the statistical dependence in network configuration, and shows that self-configuration can be optimized if the graph has nested local dependence.
\end{abstract}

\section{Introduction}

Self-configuration is for the network to achieve a desired network configuration by end-users in a distributed fashion, where each node adjusts its local configuration by following a local algorithm based on information from neighbors. A key requirement is that the local algorithm is optimal so that the desired global configuration can be achieved. The desired "globally optimal configuration" is considered to the one that makes trade-off between spatial channel-reuse maximization and constraint on both topology and reconfiguration cost. We consider topology formation and re-covery from failures. As many algorithms and protocols have provided promising results for self-configuration, it is not clear when self-configuration is implementable in a fully distributed fashion. This work intends to address the following questions: (a) How to quantify the goodness (i.e., optimality) of a network configuration? (b) When and how can local adaptation of nodes result in a globally optimal configuration?

\section{Problem Formulation}

Consider an ad-hoc wireless network with $N$ nodes, with positions, $\underline{X}=$ $\left\{X_{1}, \cdots, X_{N}\right\}$. Let $\sigma_{i j}$ be random activity of link $(i, j)$, referred to as a "communication dipole," $\sigma_{i j}=1$ if node $i$ is transmitting to node $j$; and $\sigma_{i j}=-1$, otherwise. A network configuration is a combination of both the topology and link activities, $\mathrm{G}=(\underline{\sigma}, \underline{X})$; and an optimal configuration includes an optimal physical topology $(\underline{X})$ with maximal channel reuse $(\underline{\sigma})$ under management constraints. The management constraints considered in this work are for (a) link quality (e.g. on signal to interference + noise $(\mathrm{SINR})$ ), (b) maximized geographical coverage of nodes while maintaining 1-connectivity, and (c) minimized cost in re-adjusting positions for failure recovery. The optimal configuration $\left(\underline{\sigma}^{*}, \underline{X}^{*}\right)$ is defined as 
the most likely configuration that maximizes the likelihood function, arg $\max _{(\underline{\sigma}, \underline{X})}$ $P\left(\underline{\sigma}, \underline{X} \mid \underline{\sigma_{0}}, \underline{X_{0}}\right)$, where $\left(\underline{\sigma_{0}}, \underline{X_{0}}\right)$ is the initial configuration.

Self-organization is to obtain optimal distributed algorithms characterized by local rules, $g_{i}()$, for $1 \leq i \leq N$, so that node positions can be adapted by end-users,

$$
\left(\hat{X}_{i}(t+1), \hat{\sigma_{i j}}(t+1)\right)=\underset{\left(X_{i}(t+1), \sigma_{i j}(t+1)\right)}{\arg \max } g_{i}\left(X_{i}(t+1), \sigma_{i j}(t+1) \mid X_{N_{i}}(t), \sigma_{N_{i}}(t)\right),
$$

where $N_{i}$ and $\sigma_{N_{i}}$ are the neighbors of node $i$ and dipole $\sigma_{i j}$.

\section{Cross-Layer Model of Network Configuration}

To quantify the goodness of a network configuration, we obtain the likelihood $P\left(\underline{\sigma}, \underline{X} \mid \underline{\sigma_{0}}, \underline{X_{0}}\right)$ which is from $P\left(\underline{\sigma} \mid \underline{X}, \underline{\sigma_{0}}, \underline{X_{0}}\right)$ and $P\left(\underline{X} \mid \underline{\sigma_{0}}, \underline{X_{0}}\right)$.

\subsection{Link Activities}

Assuming the traffic demand is all-to-all for simplicity, the management constraint for $\underline{\sigma}$ is then to maximize the spatial channel reuse. Thus, $P\left(\underline{\sigma} \mid \underline{X}, \underline{\sigma_{0}}, \underline{X_{0}}\right)$ $=P(\underline{\sigma} \mid \underline{X})$. Each feasible communication configuration of a network can be represented with the total energy of the network, which is referred to as "configuration Hamiltonian."

- Configuration Hamiltonian : The configuration Hamiltonian of a network is then characterized by the total negative power $-\sum_{i j} P_{j}$ and denoted as $U^{\prime}(\underline{\sigma} \mid \underline{X})$, which is $-\sum_{i j} P_{i} l_{i j}^{-4} \eta_{i j}+\sum_{i j} \sum_{m n \in N_{i j}^{I}}\left(2 \sqrt{P_{i} P_{m}} l_{i j}^{-2} l_{m j}^{-2}-P_{m} l_{m j}^{-4}\right) \eta_{i j} \eta_{m n}-$ $\sum_{i j} \sum_{m n \in N_{i j}^{I}} \sum_{u v \in\left\{N_{i j}^{I}, N_{m n}^{I}\right\}} 2 \sqrt{P_{m} P_{u}} l_{m j}^{-2} l_{u j}^{-2} \eta_{i j} \eta_{m n} \eta_{u v}+R_{I}(\underline{\sigma}, \underline{X})+R_{3}(\underline{\sigma}, \underline{X})+\beta \cdot \sum_{i j}$ $\left\|\mathrm{SINR}_{i j}-\mathrm{SINR}_{t h}\right\|$, where $\eta_{i j}=\left(\sigma_{i j}+1\right) / 2, N_{i j}^{M}$ is the neighboring dipoles within MAC range of an active dipole $\sigma_{i j}$; and the dipoles outside the MAC range, denoted as $N_{i j}^{I}$, are allowed to be active concurrently, resulting in interference. Relevant interference neighbors are those whose power exceeds a threshold, i.e., $P_{t h} \leq P_{m} \cdot l_{m j}^{-4}$. The minimum region that covers all relevant interference neighbors is referred to as the "interference range."

The interference outside the inference range is denoted with a remainder $R_{I_{i j}}(\underline{\sigma}, \underline{X})$. With $R_{I}(\underline{\sigma}, \underline{X})=\sum_{i j} R_{I_{i j}}(\underline{\sigma}, \underline{X})$, being the total contribution due to interference outside the interference range.

- Boltzmann Distribution : The total energy $U^{\prime}(\underline{\sigma} \mid \underline{X})$ can now be related to probability $P(\underline{\sigma} \mid \underline{X})$ using an analogy between communication activities in ad-hoc wireless networks and particles in statistical physics [2]. The probability distribution of particle systems obeys the Maxwell-Boltzmann distribution [2]. As a result, for a configuration Hamiltonian, the corresponding Boltzmann distribution is $P(\underline{\sigma} \mid \underline{X})=\exp \frac{-U^{\prime}(\underline{\underline{\sigma}} \mid \underline{X})}{T} / \sum_{\underline{\sigma}} \exp \frac{-U^{\prime}(\underline{\underline{\sigma}} \mid \underline{X})}{T}$. 


\subsection{Random Position of Nodes}

We now obtain the probability distribution of node positions $\mathrm{P}\left(\underline{X} \mid \underline{\sigma_{0}}, \underline{X_{0}}\right)$, assuming that current node positions are conditionally independent of initial conditions, i.e., $P\left(\underline{X} \mid \underline{\sigma_{0}}, \underline{X_{0}}\right)=P\left(\underline{X} \mid \underline{X_{0}}\right)$.

With no management purposes, nodes' movement can be characterized by a two-dimensional random-walk around fixed positions, where $P\left(\underline{\left.X \mid X_{0}\right)}\right.$ is a multivariate Gaussian distribution with an exponent $U\left(\underline{X} \mid \underline{X_{0}}\right)=\left(\underline{X}-\underline{X_{0}}\right)^{T} \cdot\left(\underline{X}-\underline{X_{0}}\right) /$ $2 \sigma^{2}$, and variance of node movement $\sigma^{2}$. The Hamiltonian $U\left(X \mid X_{0}\right)$ shows that aimless motions are penalized.

Management constraints make nodes move cooperatively to achieve a pre-defined constraint, e.g., 1-connected topology. The 1-connectivity can be achieved by a Yao-like graph [3], which can be implemented with

$$
C\left(X_{i}, X_{j}\right)= \begin{cases}0 & , \frac{\left|l_{i j}-l_{t h}\right|}{l_{t h}}<\epsilon_{0} \text { or } j \notin N_{i} \\ \left|l_{i j}-l_{t h}\right| & \text { otherwise }\end{cases}
$$

where $\epsilon_{0}$ is a small constant, $l_{t h}$ is a threshold, $l_{i j}$ is the distance between nodes $i$ and $j$.

The extended Hamiltonian for the topology is $U^{\prime}\left(\underline{X} \mid \underline{X_{0}}\right)=U\left(\underline{X} \mid \underline{X_{0}}\right)+$ $\sum_{i} \sum_{j \in N_{i}} \zeta \cdot C\left(X_{i}, X_{j}\right)$, where $U\left(\underline{X} \mid \underline{X_{0}}\right)$ is due to free movements, $\zeta$ is a weighting constant, and $N_{i}$ is the set of the nearest neighboring nodes of node $i$ for every angle $\theta\left(\theta=90^{\circ}\right.$ in this work).

For faults recovery, the propagation of configuration changes may need to be minimized across the entire network, and thus an additional penalty function can be introduced as the cost of reconfiguration, e.g., $f_{P_{1}}(\underline{\sigma}, \underline{X})=\left\|(\underline{\sigma}, \underline{X})-\left(\underline{\sigma_{0}}, \underline{X_{0}}\right)\right\|$, where \|\| denotes a vector norm. The resulting topology constraint is then $C^{\prime}\left(X_{i}, X_{j}\right)=C\left(X_{i}, X_{j}\right)+C_{1} \cdot f_{P_{1}}(\underline{\sigma}, \underline{X})$, where $C_{1}$ is constant, weighting the tradeoff between global optimization and local recovery.

\subsection{Graphical Representation of Network Configuration}

To determine the optimality of distributed self-configuration, it suffices to examine with graphical models [1] whether the obtained likelihood function is factorizable. A set of random variables, e.g., $\underline{X}$, is called a Gibbs Random Field (GRF) if it obeys Gibbs distribution, $Z_{0}^{-1} \exp \frac{-U(\underline{X})}{T}$ [1]. Hammersley-Clifford Theorem [1] shows that a GRF is is equivalent with an MRF, which shows the spatial Markov dependence among nodes.

Since the Boltzmann distribution of a network configuration obeys a Gibbs form, a network configuration can be modeled with an MRF. First, consider the random link activities given node positions. Since link activities for dipoles are all dependent, the graph is fully connected. However, $R_{I}(\underline{\sigma}, \underline{X})$ and $R_{3}(\underline{\sigma}, \underline{X})$ are negligible in Hamiltonian. The remaining two terms define an approximation $P^{l}(\underline{\sigma} \mid \underline{X})$, to the original Boltzmann distribution, where $P^{l}(\underline{\sigma} \mid \underline{X})=\frac{1}{Z_{0}}$. $\exp \left(-\sum_{i j} \alpha_{i j}(\underline{X}) \cdot \frac{\sigma_{i j}+1}{2}-\sum_{m n \in N_{i j}^{I}} \alpha_{i j, m n}(\underline{X}) \cdot \frac{\sigma_{m n}+1}{2} \frac{\sigma_{i j}+1}{2}\right) / T$. The approximated $\mathrm{MRF},(\underline{\sigma} \mid \underline{X})$, is now the well-known second-order Ising model [1]. 
Next, for the 1-connectivity topology, $U^{\prime}\left(\underline{X} \mid \underline{X_{0}}\right)$ shows that the random field, $\left(X \mid X_{0}\right)$, is a Potts model with random bonds [1] with the first-order neighbors. As a result, the coupled MRF $\left(\underline{\sigma}, \underline{X} \mid \underline{\sigma_{0}}, \underline{X_{0}}\right)$ can be fully described by an Ising model and a Potts model together, representing the nested local dependence with random bonds. Especially, for the special case of free node-movement, the coupled random field becomes a Random Bond Ising Model (RBIM) [1].

\section{Self-configuration: Distributed Algorithm and Failure-Recovery}

The nearly optimal configuration is the one that maximizes the approximated likelihood function. Maximizing the global likelihood function reduces to maximizing local likelihood at cliques, i.e., for $1 \leq i, j \leq N,\left(\hat{X}_{i}(t+1), \sigma_{i j}(t+1)\right)=$ $\arg \max _{\left(X_{i}(t+1), \sigma_{i j}(t+1)\right)} P^{l}\left(X_{i}(t+1), \sigma_{i j}(t+1) \mid X_{N_{i}}(t), \sigma_{N_{i}}(t)\right)$. Therefore, the nearly optimal local rule $g_{i}()=P^{l}()$. Stochastic relaxation is an optimal distributed algorithm through Gibbs sampling [1]. Now self configuration is viewed as local optimizations of Hamiltonian on cliques.

An important application of self-configuration is adaptive recovery from failures. Consider node failures from an optimal configuration. There is a tradeoff between global optimization of network configuration and failure localization. Figure 1 (a) shows the self-configured topology, where the failed nodes are

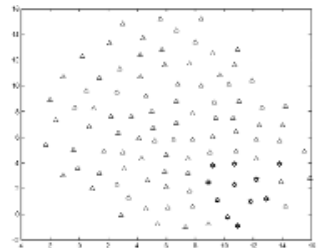

(a) Self-configuration and Failure Event (b) After Self-Recovery of $\underline{X}$

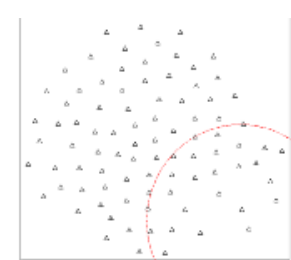

Fig. 1. Self-Recovery with Local Optimal Algorithm

marked by stars. Due to failure localization, Figure 1 (b) shows that only nodes within the arc are involved in the recovery.

\section{Conclusion}

We find that a random-bond model is the probability distribution of the network configuration where link activities are coupled by random coefficients due to node positions. Probability graphs show the conditions on when distributed selfconfiguration is optimal: (a) interference from far away nodes is negligible, and (b) management constraints are local on both connectivity and reconfiguration cost. 
Acknowledgement. This work is supported by NSF ECS-0300605 (ECS9908578). The authors would like to thank Mo Li, John Barry and Guanglei Liu for many useful discussions.

\section{References}

1. S. Geman, and D. Geman, Stochastic Relaxation, Gibbs Distributions, and the Bayesian Restoration of Images. IEEE Trans. PAMI vol. 6, 1984.

2. K. Huang, Statistical Mechanics. John Wiley \& Sons.

3. R. Wattenhofer, L. Li, P. Bahl, and Y. Wang, Distributed topology control for power efficient operation in multihop wireless networks. In Proc. IEEE Infocom, 2001 